\title{
Evaluation numérique des facteurs de forme
}

\author{
G. Ritoux \\ Laboratoire de Physique des Ondes, Université de Clermont-Ferrand, B.P. 45, 63170 Aubière, France
}

(Reçu le 17 février 1982, révisé le 13 mai 1982, accepté le 14 mai 1982)

\begin{abstract}
Résumé. - L'évaluation des facteurs de forme, liés aux transferts de chaleur par rayonnement, entre deux surfaces, se fait traditionnellement à l'aide d'abaques.

Dans ce travail, nous voulons, dans les cas pratiques les plus intéressants, permettre une évaluation rapide et précise de ces facteurs, à partir des expressions analytiques mises sous une forme facile à utiliser sur les petites calculatrices programmables courantes.
\end{abstract}

\begin{abstract}
The form factors used in the study of heat radiation transfer between two surfaces are generally evaluated with nomograms.

In this paper, we want to derive in the most important practical cases, an exact and rapid evaluation of these factors, from the analytic expressions written in a form easy to program with usual little computers.
\end{abstract}

Les facteurs de forme de surfaces quelconques, utilisés dans les études de transfert d'énergie par rayonnement, sont en général fournis par des abaques. En effet, leur détermination, mis à part quelques rares systèmes à géométrie simple, est généralement très pénible, et les chercheurs préfèrent utiliser des abaques quand ils existent, plutôt que de chercher des solutions analytiques.

Or, il est possible, dans plusieurs cas pratiques importants d'obtenir ces solutions analytiques par intégration sur les surfaces étudiées. De plus, les solutions obtenues peuvent être mises sous une forme telle qu'elles deviennent très simples à programmer sur les petites calculatrices modernes.

Les solutions analytiques présentent de nombreux avantages : précision, domaine d'application non limité, études de systèmes centrés ou non, etc... Elles permettent surtout de résoudre les cas limites, ce qui n'est pas possible avec les abaques. Nous nous sommes ainsi particulièrement intéressés à un cas pratique important, celui d'une surface émettrice élémentaire de petites dimensions devant celles des surfaces réceptrices et ce, quelle que soit sa position relative visà-vis d'elles. Ce problème était d'autant plus intéressant à résoudre que seuls quelques cas très particuliers paraissent résolus numériquement.

Nous étudierons successivement dans ce travail, les facteurs de forme des surfaces rectangulaires planes, puis des dièdres droits, enfin des surfaces circulaires planes, toutes les expressions analytiques étant systématiquement mises sous une forme facile à programmer.

1. Surfaces planes rectangulaires (ou carrées). - 1.1 SuRfaCES RECTANGULAIRES PLANES ET Parallèles, DE DIMENSIONS QUELCONQUES, NON CENTRÉES.

$$
\begin{aligned}
\mathbf{M}^{\prime} \mathbf{M} & =\mathbf{r} \\
\mathbf{O}^{\prime} \mathbf{M}^{\prime} & =\boldsymbol{\rho}^{\prime}\left(X^{\prime}, Y^{\prime}, 0\right) \\
\mathbf{O M} & =\boldsymbol{\rho}(X, Y, 0) \\
\mathbf{O}^{\prime} \mathbf{O}^{\prime \prime} & =d
\end{aligned}
$$

$$
\begin{aligned}
\text { OO }^{\prime \prime} & =\rho_{0}\left(a_{0}, b_{0}, 0\right) \\
r^{2} & =\left(X-X^{\prime}-a_{0}\right)^{2}+\left(Y-Y^{\prime}-b_{0}\right)^{2}+d^{2} \\
\cos \theta & =\cos \theta^{\prime}=d / r .
\end{aligned}
$$




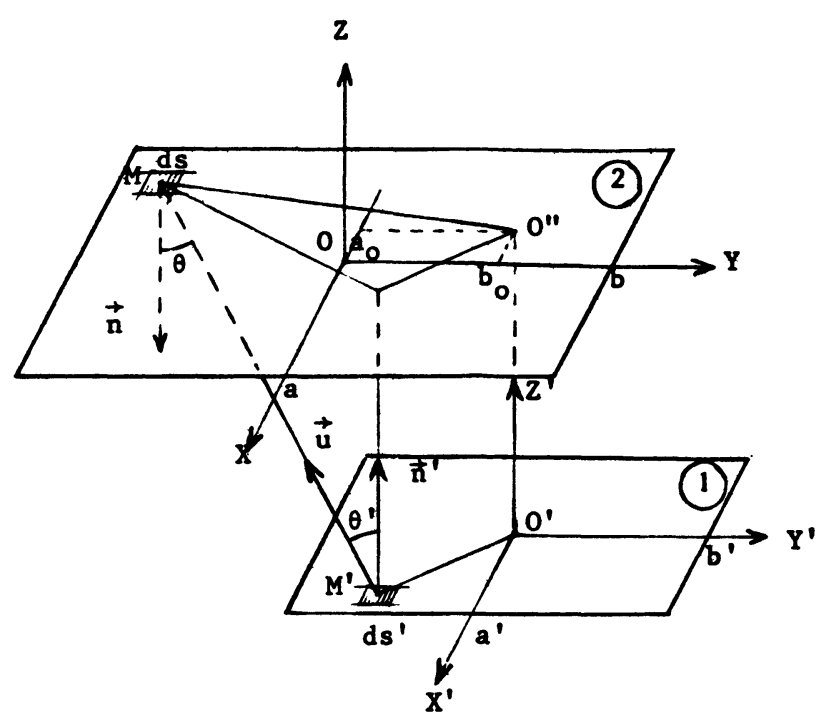

Dimensions des plaques :

$$
\begin{array}{cr}
\text { 1. }-a^{\prime}<X^{\prime}<+a^{\prime} & \text { 2. }-a<X<+a \\
-b^{\prime}<Y^{\prime}<+b^{\prime} & -b<Y<+b \\
F_{12}=\frac{1}{\pi s^{\prime}} \int_{s} \int_{s^{\prime}} \frac{\cos \theta \cos \theta^{\prime} \mathrm{d} s \mathrm{~d} s^{\prime}}{r^{2}}=\frac{d^{2}}{\pi s^{\prime}} \int_{s} \int_{s^{\prime}} \frac{\mathrm{d} s \mathrm{~d} s^{\prime}}{r^{4}} .
\end{array}
$$

Afin de pouvoir généraliser les résultats, on introduira les dimensions réduites des surfaces que l'on notera ainsi.

$$
\begin{array}{lll}
X=a / d & Y=b / d & X_{0}=a_{0} / d \\
X^{\prime}=a^{\prime} / d & Y^{\prime}=b^{\prime} / d & Y_{0}=b_{0} / d
\end{array}
$$
faits.

L'intégrale quadruple qui permet le calcul du facteur de forme de $s^{\prime}$ vers $s$ (de 1 vers 2 ) donne tous calculs

$$
\begin{aligned}
F_{12}=\frac{1}{8 \pi X^{\prime} Y^{\prime}}( & F( \\
& \left.X-X_{0}+X^{\prime}, Y-Y_{0}+Y^{\prime}\right)-F\left(X-X_{0}+X^{\prime}, Y-Y_{0}-Y^{\prime}\right)+F\left(X-X_{0}+X^{\prime}, Y+Y_{0}-Y^{\prime}\right)- \\
& -F\left(X-X_{0}+X^{\prime}, Y+Y_{0}-Y^{\prime}\right)-F\left(X-X_{0}-X^{\prime}, Y-Y_{0}+Y^{\prime}\right)+F\left(X-X_{0}-X^{\prime}, Y-Y_{0}-Y^{\prime}\right) \\
& -F\left(X-X_{0}-X^{\prime}, Y+Y_{0}-Y^{\prime}\right)+F\left(X-X_{0}-X^{\prime}, Y+Y_{0}-Y^{\prime}\right)+F\left(X+X_{0}+X^{\prime}, Y-Y_{0}+Y^{\prime}\right) \\
& -F\left(X+X_{0}+X^{\prime}, Y-Y_{0}-Y^{\prime}\right)+F\left(X+X_{0}+X^{\prime}, Y+Y_{0}-Y^{\prime}\right)-F\left(X+X_{0}+X^{\prime}, Y+Y_{0}-Y^{\prime}\right) \\
& -F\left(X+X_{0}-X^{\prime}, Y-Y_{0}+Y^{\prime}\right)+F\left(X+X_{0}-X^{\prime}, Y-Y_{0}-Y^{\prime}\right)-F\left(X+X_{0}-X^{\prime}, Y+Y_{0}-Y^{\prime}\right) \\
& \left.+F\left(X+X_{0}-X^{\prime}, Y+Y_{0}-Y^{\prime}\right)\right) .
\end{aligned}
$$

Avec

$$
F(u, v)=u \sqrt{v^{2}+1} \operatorname{Arctg} \frac{u}{\sqrt{v^{2}+1}}+v \sqrt{u^{2}+1} \operatorname{Arctg} \frac{v}{\sqrt{u^{2}+1}}-\frac{1}{2} \operatorname{Ln}\left(u^{2}+v^{2}+1\right)
$$

Cette formule complexe, mais facile à programmer, est d'une réelle importance. Elle contient, en effet, tous les cas de figures pour calculer les facteurs de forme de n'importe quelles surfaces planes et parallèles, rectangulaires ou carrées, centrées ou non. Or, toutes les références ne traitent que les surfaces centrées, ou bien les surfaces dont l'une a des dimensions petites et se trouve en position particulière vis-à-vis de l'autre et jamais de façon analytique.

Afin de bien montrer l'intérêt de cette expression, nous allons envisager plusieurs cas particuliers traités ou non dans la littérature, mais dont les facteurs de forme dérivent tous de cette formule. 
Posons $Y_{0}=0$ dans (1)

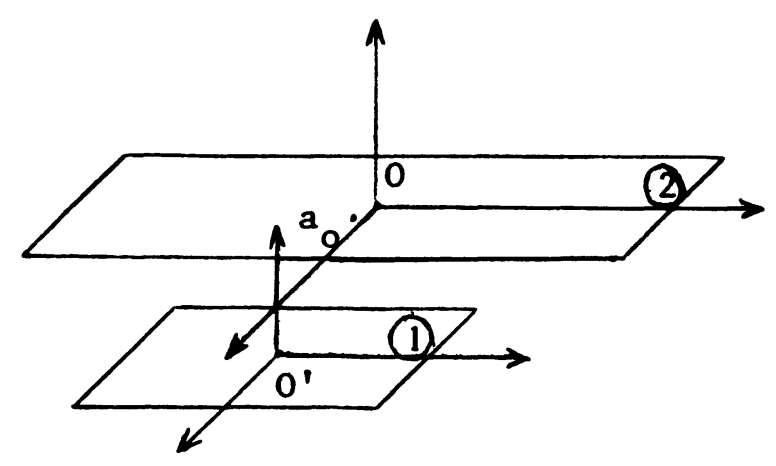

$$
\begin{array}{r}
F_{12}=\frac{1}{4 \pi X^{\prime} Y^{\prime}}\left(F\left(X-X_{0}+X^{\prime}, Y+Y^{\prime}\right)-F\left(X-X_{0}+X^{\prime}, Y-Y^{\prime}\right)-F\left(X-X_{0}-X^{\prime}, Y+Y^{\prime}\right)+\right. \\
+F\left(X-X_{0}-X^{\prime}, Y-Y^{\prime}\right)+F\left(X+X_{0}+X^{\prime}, Y+Y^{\prime}\right)-F\left(X+X_{0}+X^{\prime}, Y-Y^{\prime}\right)- \\
\left.-F\left(X+X_{0}-X^{\prime}, Y+Y^{\prime}\right)+F\left(X+X_{0}-X^{\prime}, Y-Y^{\prime}\right)\right)
\end{array}
$$

Cette formule nous donne le facteur de forme de 1 vers 2 pour deux plans rectangulaires et parallèles dont les centres sont décalés de $\pm X_{0}$ sur l'axe des $X$ (en coordonnées réduites, donc de $\pm a_{0}$ en réalité).

On peut écrire une formule analogue en faisant $X_{0}=0$ dans (1) avec des rectangles décalés suivant les $Y$ de $\pm Y_{0}$.

1.2 Surfaces Rectangulaires Planes et Parallèles, DE Dimensions QuelconQues, CentrÉEs.

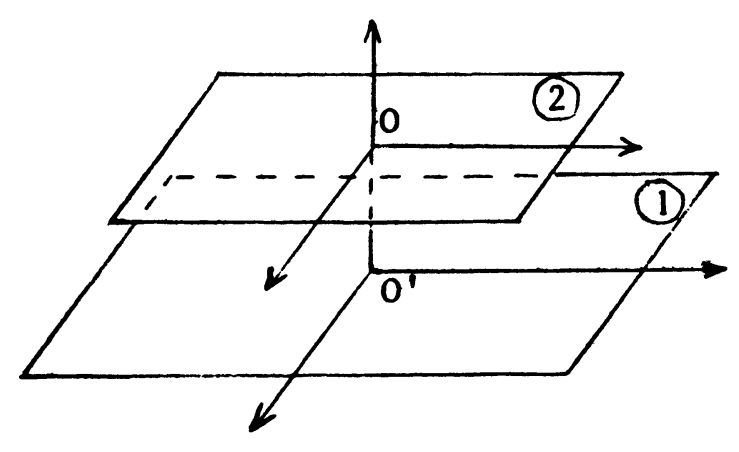

On pose $X_{0}=Y_{0}=0$ dans (1).

$$
F_{12}=\frac{1}{2 \pi X^{\prime} Y^{\prime}}\left(F\left(X+X^{\prime}, Y+Y^{\prime}\right)-F\left(X+X^{\prime}, Y-Y^{\prime}\right)-F\left(X-X^{\prime}, Y+Y^{\prime}\right)+F\left(X-X^{\prime}, Y-Y^{\prime}\right)\right)
$$

A partir de cette formule, on peut déduire plusieurs facteurs de forme dans des cas particuliers intéressants du point de vue pratique.

1) $Y \gg Y^{\prime}$

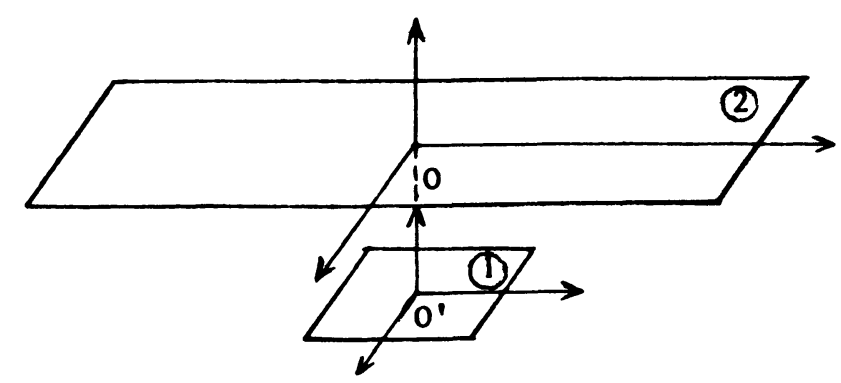


Remarquons que l'on peut écrire :

$$
F\left(u, Y+Y^{\prime}\right) \simeq F(u, Y)+Y^{\prime} F_{v}^{\prime}(u, Y)
$$

et

$$
F\left(u, Y-Y^{\prime}\right) \simeq F(u, Y)-Y^{\prime} F_{v}(u, Y)
$$

En reportant ceci en (3), il vient :

$$
F_{12}=\frac{1}{\pi X^{\prime}}\left(F_{v}^{\prime}\left(X+X^{\prime}, Y\right)-F_{v}^{\prime}\left(X-X^{\prime}, Y\right)\right)
$$

avec

$$
F_{v}^{\prime}(u, v)=\frac{u v}{\sqrt{v^{2}+1}} \operatorname{Arctg} \frac{u}{\sqrt{v^{2}+1}}+\sqrt{u^{2}+1} \operatorname{Arctg} \frac{v}{\sqrt{u^{2}+1}}
$$

On a donc ainsi le facteur de forme de deux plaques planes rectangulaires et parallèles, dont la surface réceptrice est un rectangle long et étroit.

Il est très facile par la même voie de montrer que pour l'autre dimension, on a :

2) $\underline{X \gg X^{\prime}}$

$$
F_{12}=\frac{1}{\pi Y^{\prime}}\left(F_{u}^{\prime}\left(X, Y+Y^{\prime}\right)-F_{u}^{\prime}\left(X, Y-Y^{\prime}\right)\right)
$$

avec

$$
F_{u}^{\prime}(u, v)=\sqrt{v^{2}+1} \operatorname{Arctg} \frac{u}{\sqrt{v^{2}+1}}+\frac{u v}{\sqrt{u^{2}+1}} \operatorname{Arctg} \frac{v}{\sqrt{u^{2}+1}}
$$

3) $\underline{Y^{\prime} \gg Y}$

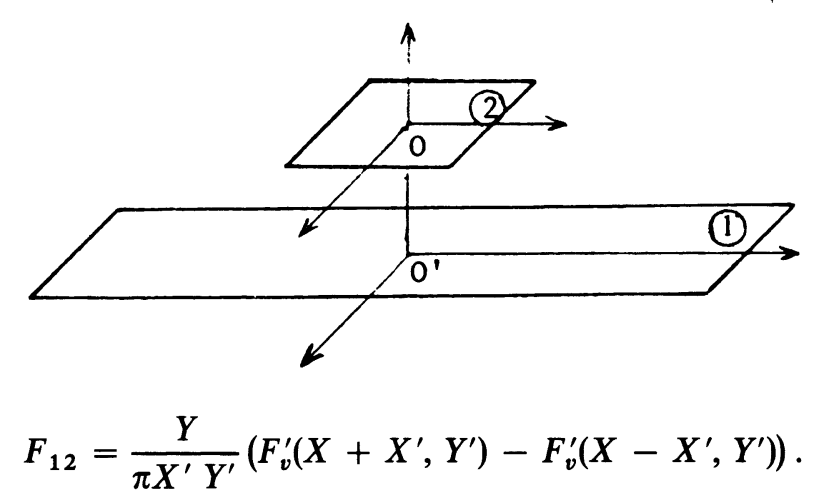

C'est cette fois l'émetteur qui est un rectangle long et étroit. Reste le cas où $X^{\prime} \gg X$.

4) $\underline{X^{\prime} \gg X}$

$$
F_{12}=\frac{X}{\pi X^{\prime} Y^{\prime}}\left(F_{u}^{\prime}\left(X^{\prime}, Y+Y^{\prime}\right)-F_{u}^{\prime}\left(X^{\prime}, Y-Y^{\prime}\right)\right)
$$

5) $\underline{Y \gg Y^{\prime} \text { et } X \gg X^{\prime}}$

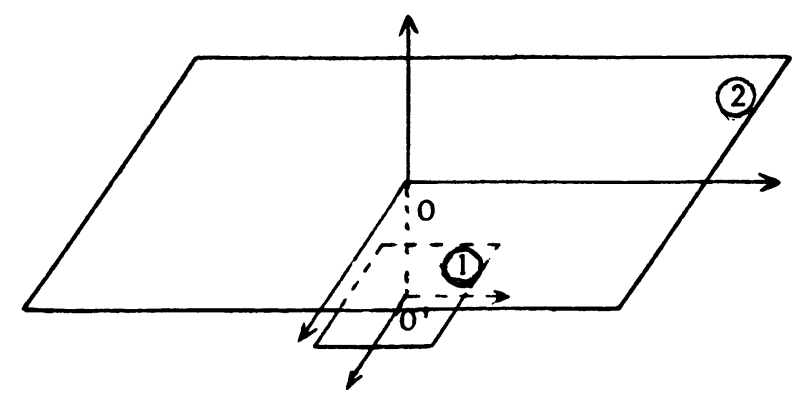

$$
F_{12}=\frac{2}{\pi} F_{u, v}^{\prime \prime}(X, Y)
$$


avec

$$
F_{u, v}^{\prime \prime}(u, v)=\frac{v}{\sqrt{v^{2}+1}} \operatorname{Arctg} \frac{u}{\sqrt{v^{2}+1}}+\frac{u}{\sqrt{u^{2}+1}} \operatorname{Arctg} \frac{v}{\sqrt{u^{2}+1}} .
$$

Le récepteur est très grand devant l'émetteur. Mais ceci est un cas particulier : celui de l'émission d'une surface élémentaire centrée par rapport au récepteur. Le cas pratique le plus courant est celui de l'émission d'une petite surface élémentaire quelconque quant à sa position.

Repérons par $a_{0}$ et $b_{0}$ les coordonnées de son centre par rapport au milieu $\mathrm{O}^{\prime}$ de la surface support. Le facteur de forme est alors donné par :

$$
F_{12}=\frac{d^{2}}{\pi} \int_{s} \frac{\mathrm{d} s}{r^{4}}
$$

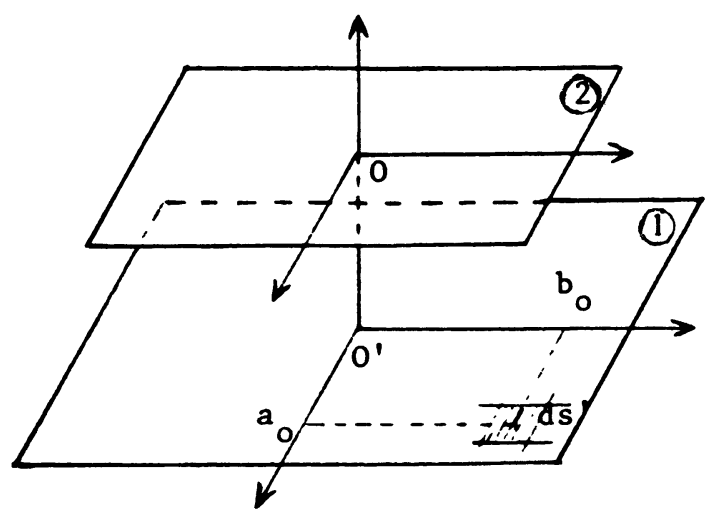

$$
\begin{array}{r}
F_{12}=\frac{1}{2 \pi}\left(F_{u v}^{\prime \prime}\left(X+X_{0}, Y+Y_{0}\right)+F_{u v}^{\prime \prime}\left(X+X_{0}, Y-Y_{0}\right)+F_{u v}^{\prime \prime}\left(X-X_{0}, Y+Y_{0}\right)+\right. \\
\left.+F_{u v}^{\prime \prime}\left(X-X_{0}, Y-Y_{0}\right)\right) .
\end{array}
$$

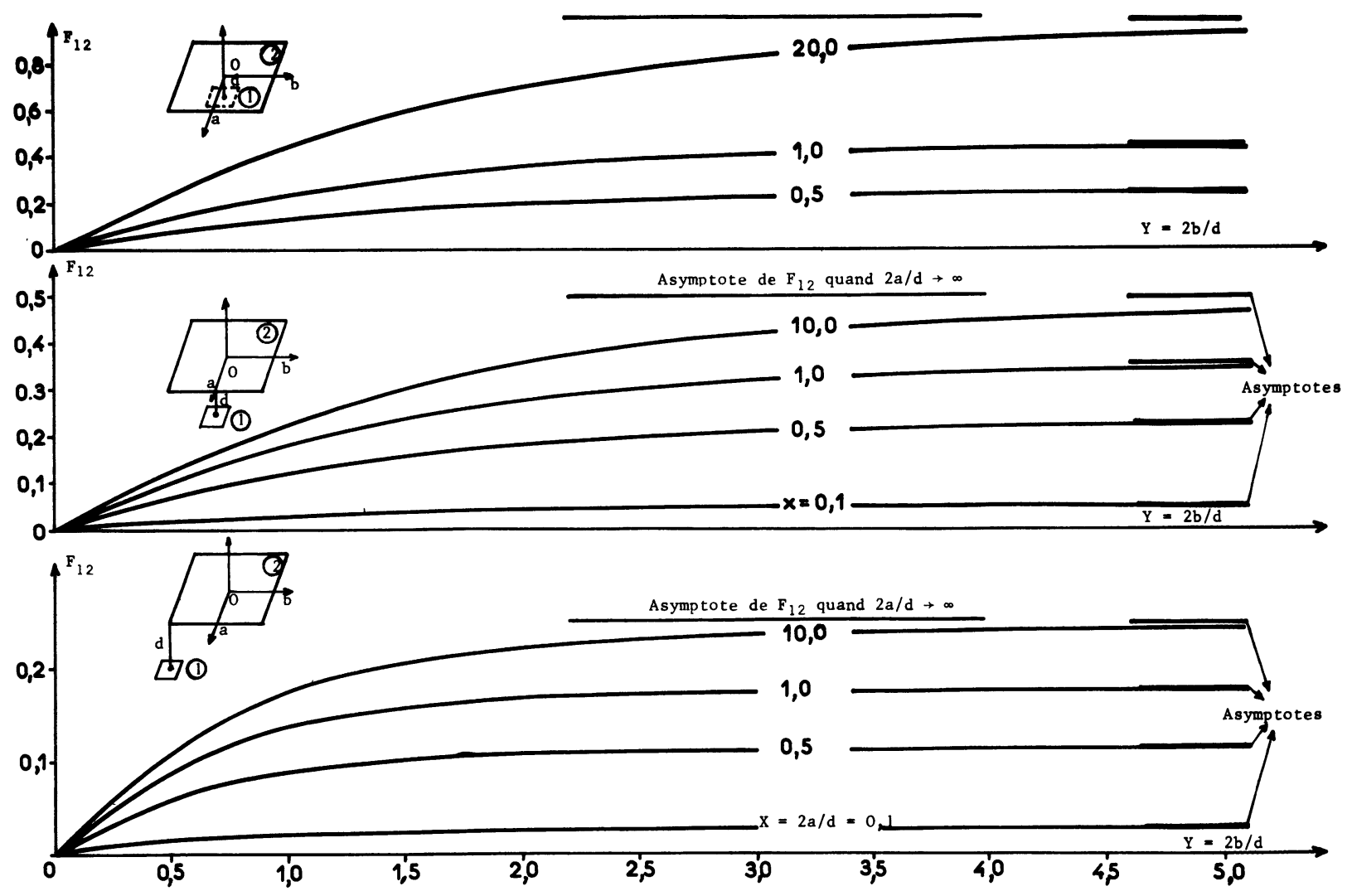

Fig. 1. - Variations du facteur de forme d'un élément de surface émettrice dans trois positions relatives différentes par rapport à la surface réceptrice parallèle, en fonction de $Y$, d'après (9).

[Variations of the form factor of an emitting surface element in three different relative positions as a function of $Y$ according to (9).] 
Il importe de remarquer que cette formule, comme les précédentes, se déduit directement de (1), ce qui démontre bien l'intérêt de l'expression générale.

Afin de vérifier les résultats obtenus, nous avons représenté sur la figure 1, les facteurs de forme d'une petite surface élémentaire dans trois positions différentes vis-à-vis de la surface réceptrice. Si la.dernière de ces positions se trouve souvent reproduite $[3,4]$ et permet une vérification facile, il n'en est pas de même pour les deux autres ni pour des petites surfaces dont les positions relatives sont quelconques.

Nota : $X_{0}=Y_{0}=0$, on retrouve (8).

6) $\underline{Y^{\prime} \gg Y \text { et } \quad X^{\prime} \gg X}$

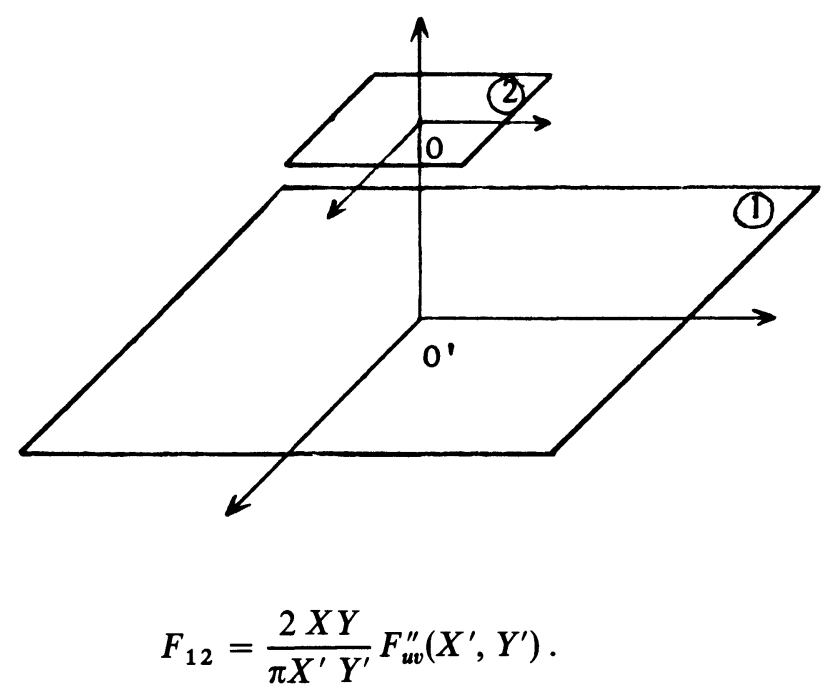

$F_{12}$ est la fraction du flux recueilli par le récepteur supposé très petit devant les dimensions de la surface émettrice.

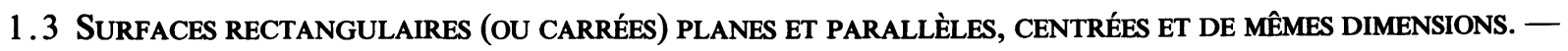
1) $X=X^{\prime}$ et $Y=Y^{\prime}$ : rectangles de mêmes dimensions.

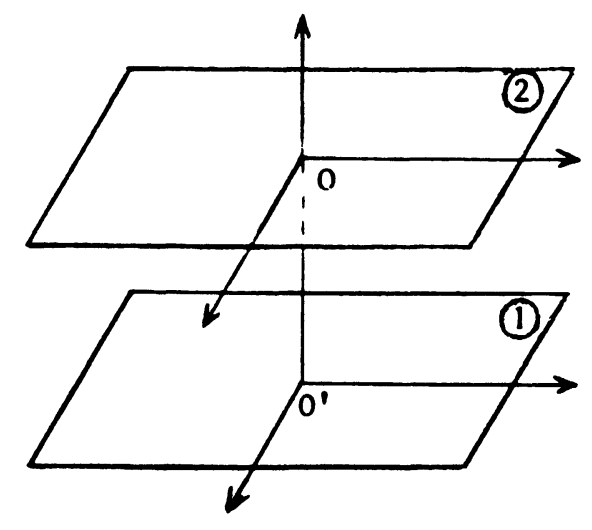

$$
F_{12}=\frac{1}{2 \pi X Y}(F(2 X, 2 Y)-F(2 X, 0)-F(0,2 Y))
$$

2) $\underline{X=X^{\prime}=Y=Y^{\prime}}$ : carrés de mêmes dimensions.

$$
F_{12}=\frac{1}{2 \pi X^{2}}(F(2 X, 2 X)-F(2 X, 0)-F(0,2 X)) .
$$


3) $\underline{Y=Y^{\prime} \gg X=X^{\prime}}$ : rectangles longs et étroits.

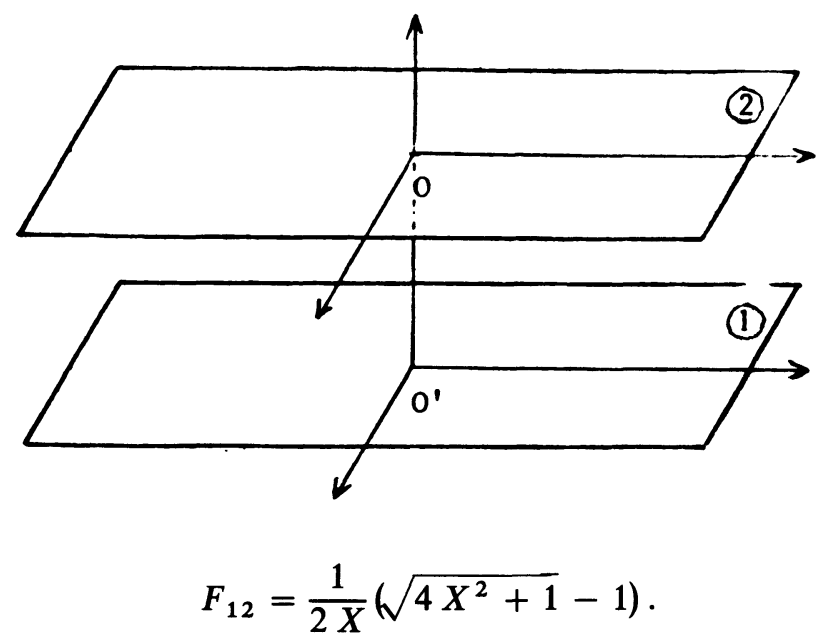

4) $X=X^{\prime}=Y=Y^{\prime} \gg 1$ : carrés de très grandes dimensions.

ou $X=X^{\prime} \neq Y=Y^{\prime} \gg 1$ : rectangles de très grandes dimensions.

$$
F_{12} \simeq 1
$$

Nous avons représenté sur la figure 2 quelques courbes donnant les facteurs de forme de deux surfaces rectangulaires égales parallèles et opposées représentatives de la formule (11). On pourra aisément vérifier ces résultats, ce système étant un des plus abondamment traité dans les ouvrages spécialisés.

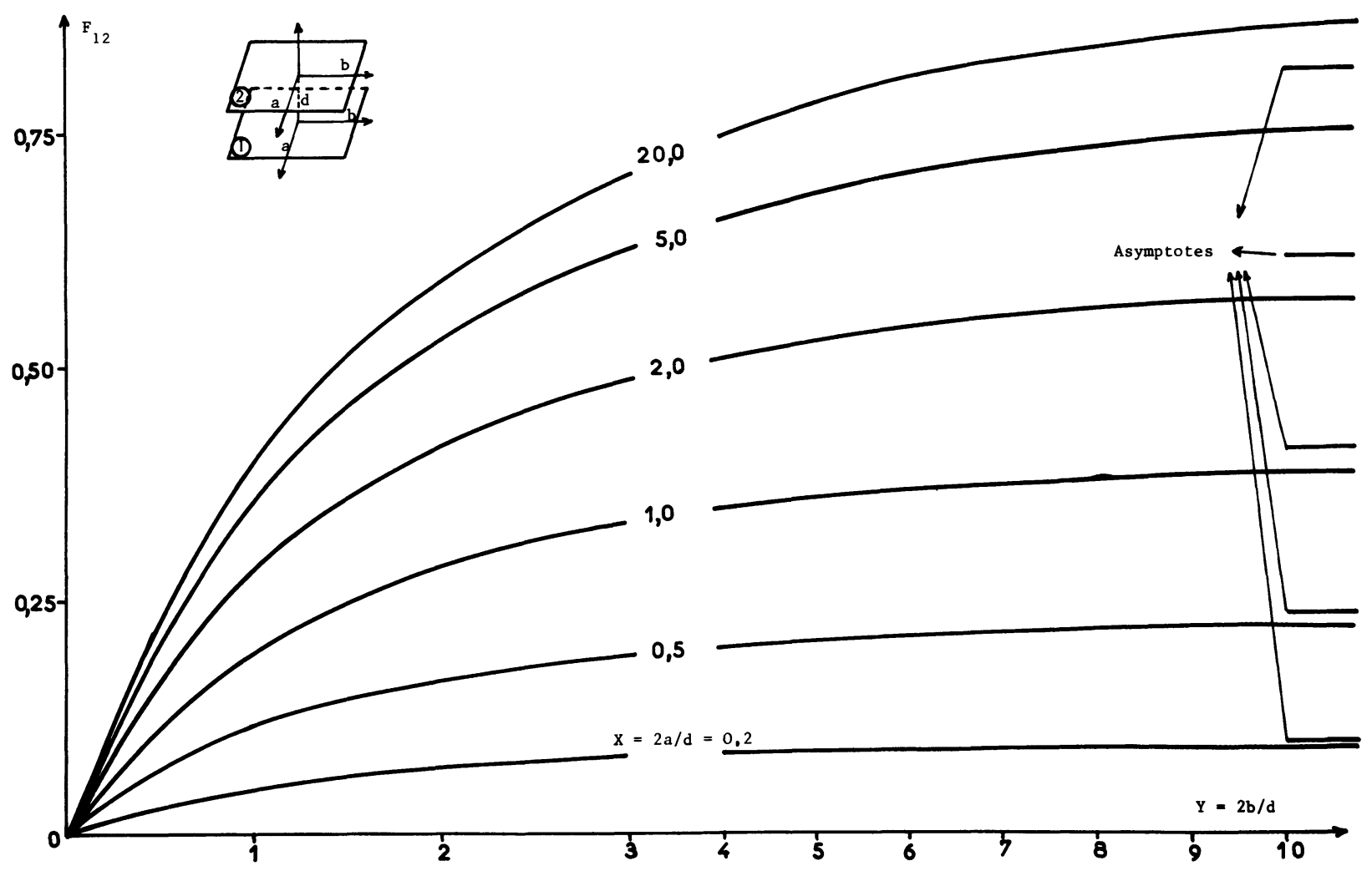

Fig. 2. - Facteurs de forme de deux surfaces rectangulaires et parallèles de mêmes dimensions, d'après (11).

[Form factors of two rectangular parallel surfaces having the same dimensions according to (11).] 
2. Surfaces planes rectangulaires formant un drièdre droit.

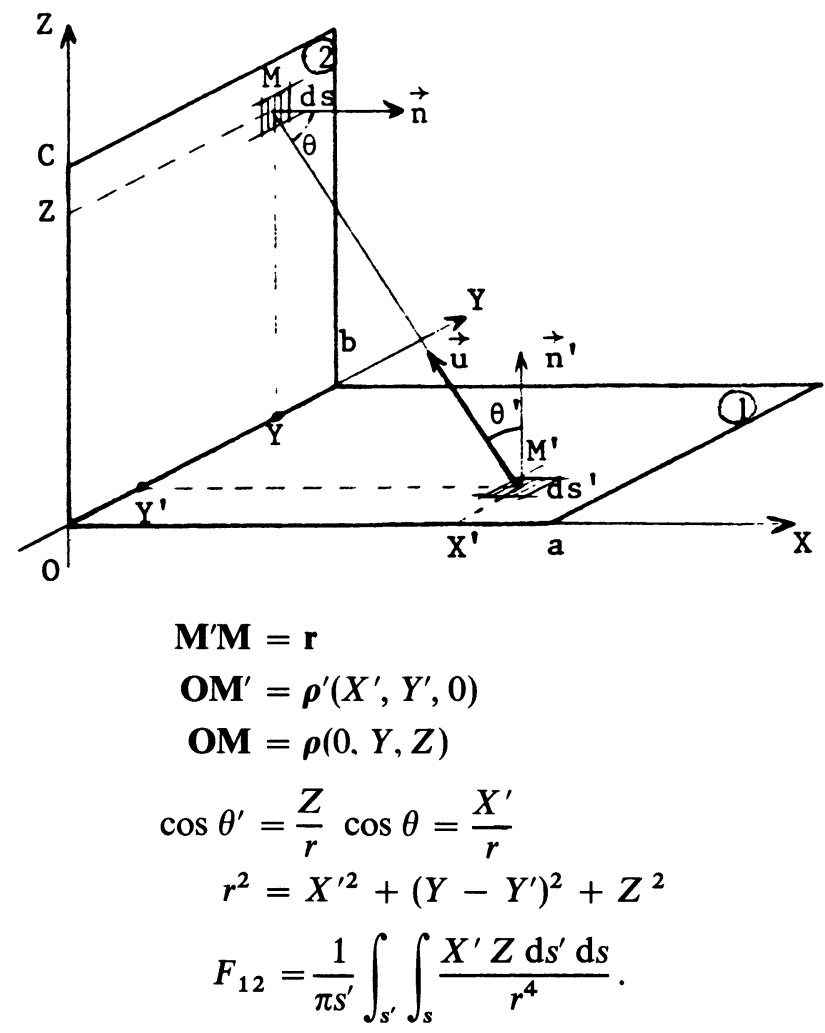

Nous introduisons les coordonnées réduites suivantes. Toutes les dimensions sont rapportées à la longueur commune aux deux rectangles $: b$.

$$
X=a / b \quad Z=c / b .
$$

L'intégrale se résout entièrement. Tous calculs faits, on trouve

$$
F_{12}=\frac{1}{4 \pi X}(F(X, Z)-F(X, 0)-F(0, Z))
$$

avec

$F(u, v)=-4 \sqrt{u^{2}+v^{2}} \operatorname{Arctg} \frac{1}{\sqrt{u^{2}+v^{2}}}+\left(u^{2}+v^{2}-1\right) \operatorname{Ln}\left(1+u^{2}+v^{2}\right)-\left(u^{2}+v^{2}\right) \operatorname{Ln}\left(u^{2}+v^{2}\right)$

Comme pour les surfaces planes, rectangulaires et parallèles, c'est cette première formule qui est importante, puisque c'est à partir d'elle que l'on déduira les cas particuliers que l'on aura éventuellement à traiter A titre d'exemple, nous avons choisi deux cas limites intéressants, non traités à notre connaissance et qui illustrent bien les possibilités des expressions analytiques. Enfin, nous donnons l'expression du facteur de forme pour une petite surface émettrice en position quelconque, système également ignoré même numériquement.

Deux cas limites sont intéressants dans la pratique.

$2.1 Z \gg 1$. - La surface réceptrice devient un rectangle très long de base constante $b$.

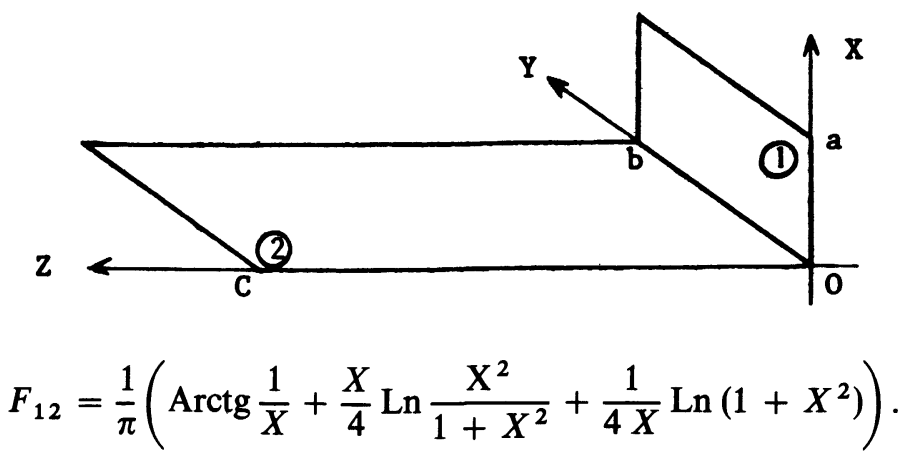


$2.2 X \ll 1 .-$ Alors

$$
F_{12}=\frac{1}{2}
$$

Un cas également intéressant est celui de l'émission d'une petite surface élémentaire en position quelconque sur la surface $s^{\prime}$.

2.3 FACTEUR DE FORME D'UNE PETITE SURFACE EN POSITION QUELCONQUE.

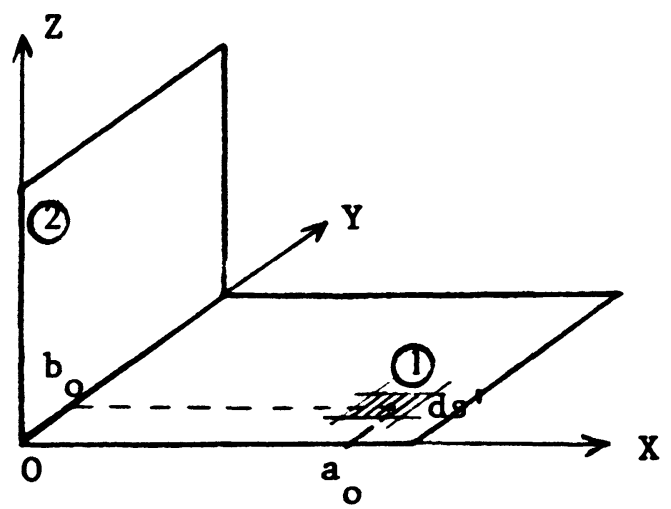

Repérons par $a_{0}$ et $b_{0}$ les coordonnées de son centre, soit $X_{0}$ et $Y_{0}$ en coordonnées réduites telles que :

$$
X_{0}=a_{0} / b \text { et } Y_{0}=b_{0} / b \text {. }
$$

Le facteur de forme de 1 vers 2 est donné par :

$$
F_{12}=\frac{X^{\prime}}{\pi} \int_{s} \frac{Z}{r^{4}} \mathrm{~d} s .
$$

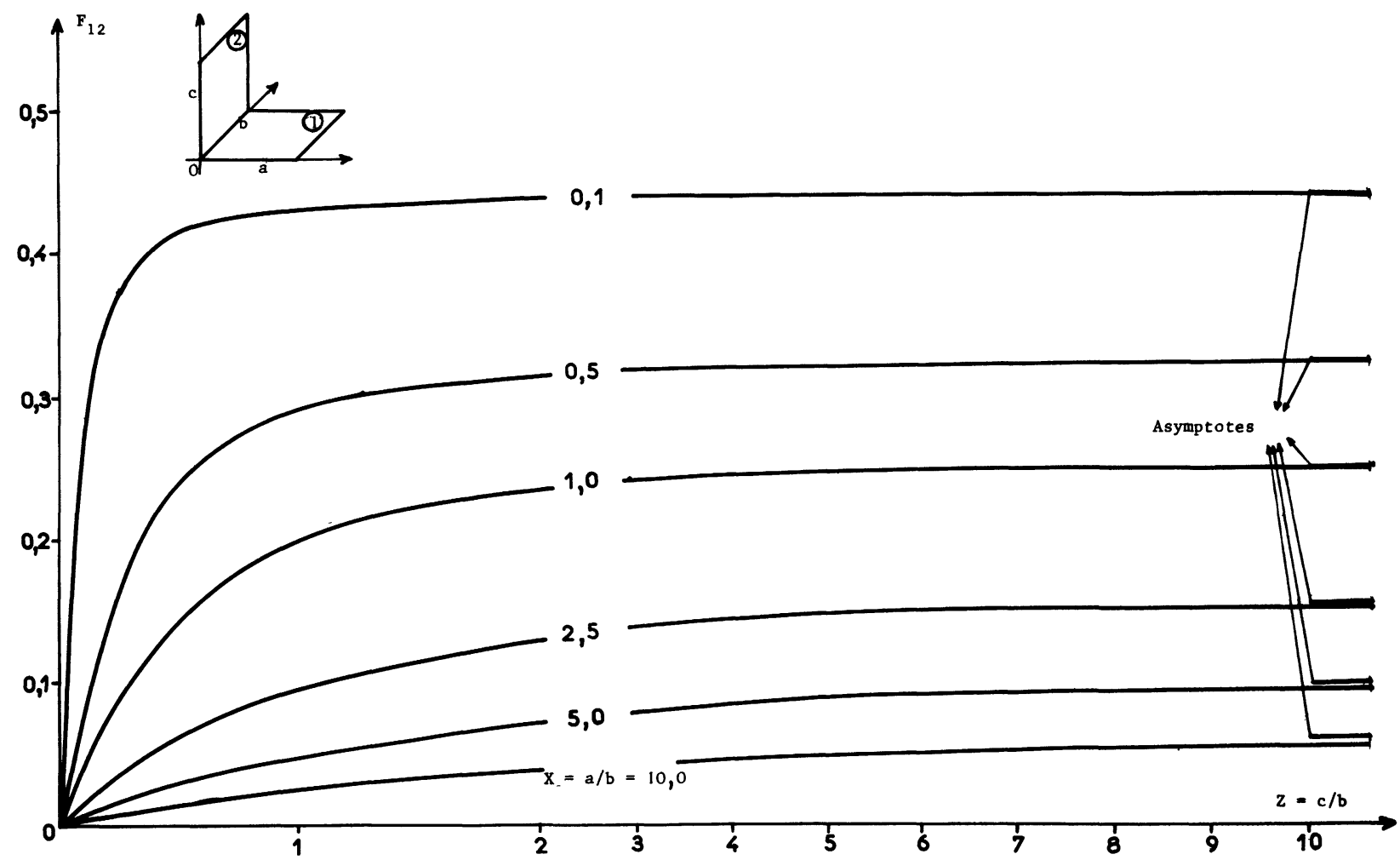

Fig. 3. - Facteurs de forme de surfaces rectangulaires formant un dièdre droit, en fonction de la hauteur réduite $Z$ de la surface réceptrice, d'après $(15)$.

[Form factors of rectangular surfaces making a rectangular dihedron as a function of the reduced height $Z$, of the receiving surface according to (15).] 
Tous calculs faits, il vient :

$$
F_{12}=\frac{X_{0}}{2 \pi}(G(0)-G(Z))
$$

avec

$$
G(u)=\frac{1}{\sqrt{X_{0}^{2}+u^{2}}}\left(\operatorname{Arctg} \frac{1-Y_{0}}{\sqrt{u^{2}+X_{0}^{2}}}+\operatorname{Arctg} \frac{Y_{0}}{\sqrt{u^{2}+X_{0}^{2}}}\right) .
$$

La figure 3 nous montre l'allure des facteurs de forme de deux surfaces rectangulaires formant un dièdre droit, en fonction de la hauteur réduite $Z$, de la surface réceptrice et de la largeur réduite $X$, de la surface émettrice. On notera que $F_{12}$ est d'autant plus grand que $X$ est plus petit, ce qui se comprend aisément. Nous avons choisi ce système car il permet une vérification aisée. En effet, il est traité à peu près dans tous les livres spécialisés.

La figure 4 représente les variations du facteur de forme d'une petite surface élémentaire vers une surface réceptrice normale à son plan support, pour deux positions relatives différentes des surfaces, conformément à la formule (18). Notons que l'on ne trouve pas ce cas dans la littérature bien qu'il présente à notre avis un intérêt certain.

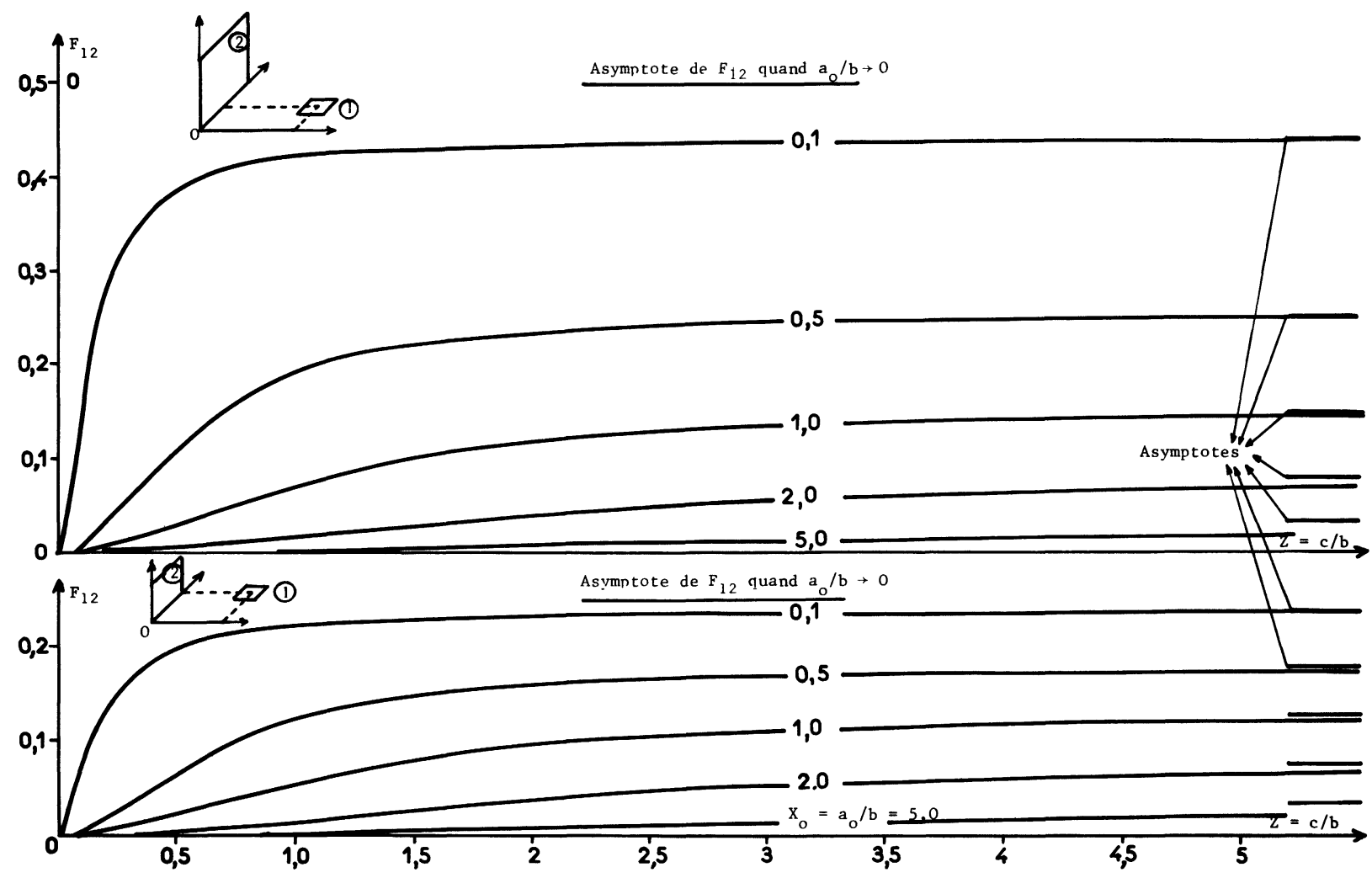

Fig. 4. - Variations du facteur de forme d'un élément de surface émettrice vers une surface réceptrice normale, pour deux positions relatives différentes d'après (18).

[Variations of the form factor of an emetting surface element towards the normal receiving surface in two different relative positions according to (18).]

\section{Surfaces planes circulaires.}

$$
\begin{aligned}
\mathbf{M}^{\prime} \mathbf{M} & =\mathbf{r} \\
\mathbf{O M} & =\boldsymbol{\rho}(\rho, \phi, d) \\
\mathbf{O M}^{\prime} & =\boldsymbol{\rho}^{\prime}\left(\rho^{\prime}, \phi^{\prime}, 0\right) \\
r^{2} & =\rho^{2}+\rho^{\prime 2}-2 \rho \rho^{\prime} \cos \left(\phi-\phi^{\prime}\right)+d^{2} \\
\cos \theta= & \cos \theta^{\prime}=d / r \\
& F_{12}=\frac{d^{2}}{\pi s^{\prime}} \int_{s} \int_{s^{\prime}} \frac{\mathrm{d} s \mathrm{~d} s^{\prime}}{r^{4}}
\end{aligned}
$$




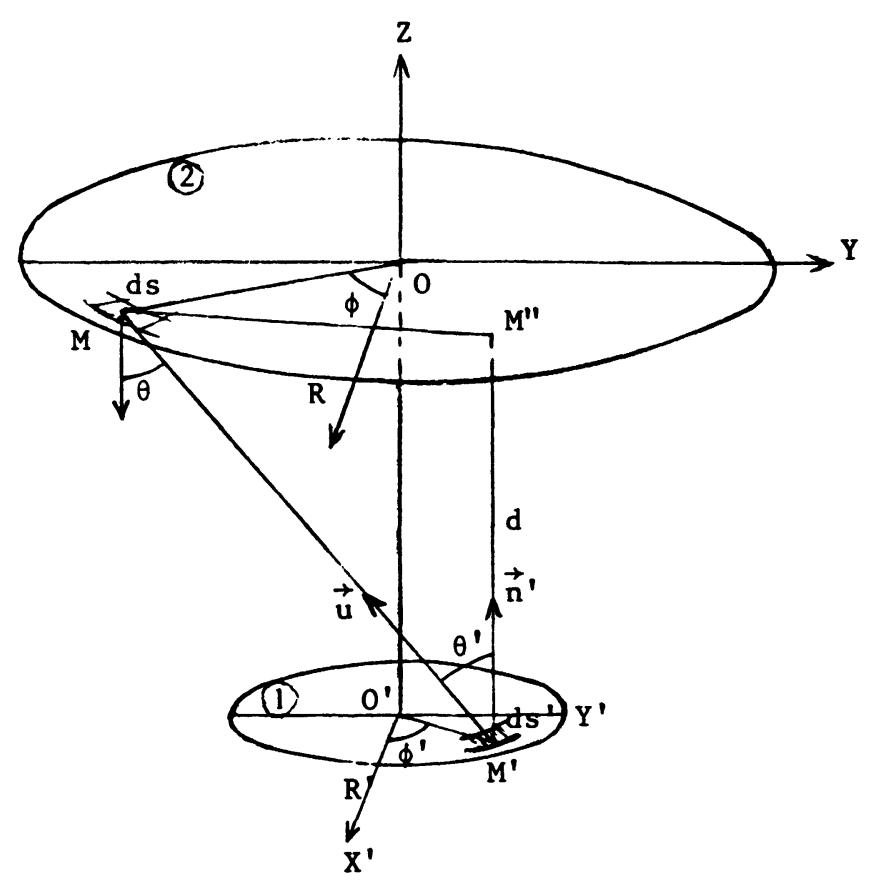

Coordonnées réduites :

$$
X=R / d \quad X^{\prime}=R^{\prime} / d
$$

L'intégration de $F_{12}$ donne un résultat connu. Il s'écrit :

$$
F_{12}=\frac{1}{2 X^{\prime 2}}\left(1+X^{2}+X^{\prime 2}-\sqrt{\left(1+X^{2}+X^{\prime 2}\right)^{2}-4 X^{2} X^{\prime 2}}\right) .
$$

La représentation graphique de cette formule, donne des courbes tout à fait comparables à celles obtenues figure 2 pour des surfaces rectangulaires. Ajoutons que cette expression est pratiquement la seule expression analytique que l'on trouve dans la littérature [3].

Des cas limites intéressants peuvent s'en déduire :

$3.1 R \rightarrow \infty$ ou $X \gg 1$

$$
F_{12} \simeq 1-\frac{X^{\prime 2}}{X^{2}}
$$

$3.2 R^{\prime} \rightarrow 0$ ou $X^{\prime} \ll 1$

$$
F_{12}=\frac{X^{2}}{1+X^{2}}
$$

On peut dans ce cas se poser le problème de l'émission d'une petite surface quelconque quant à sa position. Le système possédant la symétrie de révolution autour de l'axe $Z$, il est évident que le facteur de forme ne dépend que du rayon $\rho_{0}$ et pas de $\phi_{0}$.

\subsection{FACTEUR DE FORME D'UNE PETITE SURFACE EN POSITION QUELCONQUE. - Il sera donné par l'intégrale}

$$
F_{12}=\frac{d^{2}}{\pi} \int \frac{\mathrm{d} s}{r^{4}}
$$

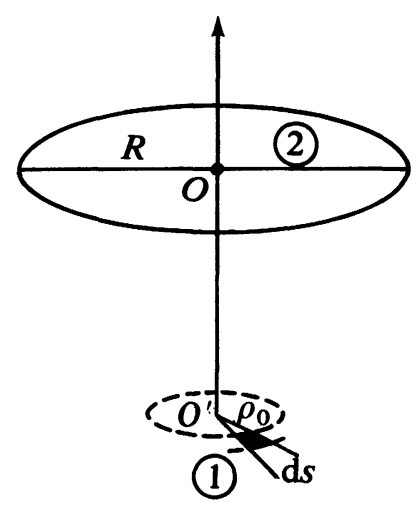




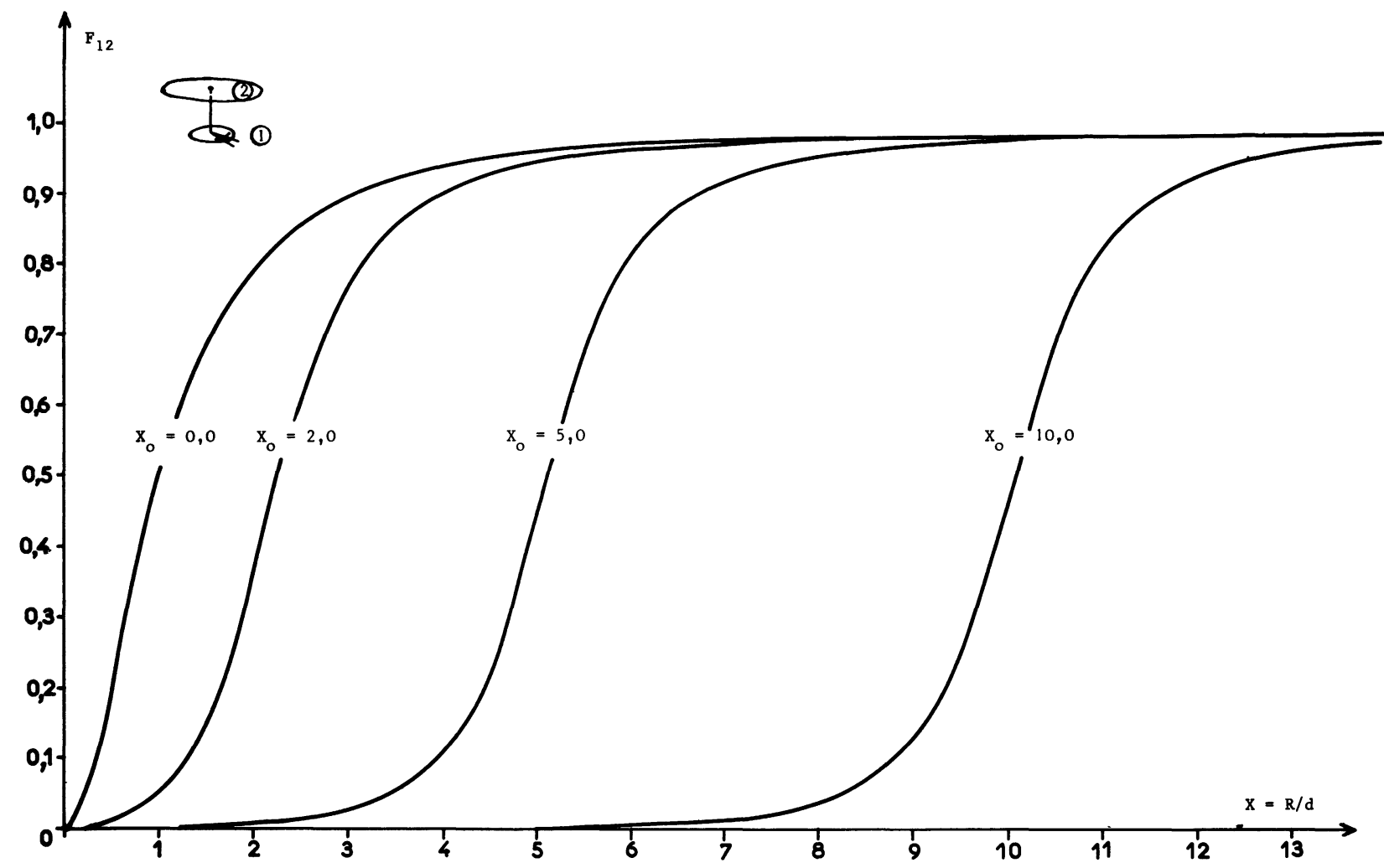

Fig. 5. - Variations du facteur de forme d'un élément de surface émettrice vers une surface circulaire, en fonction du rayon réduit, d'après (22).

[Variations of the form factors of an emitting surface element towards the receiving circular surface as a function of the reduced radius according (22).]

Nous poserons en coordonnées réduites :

$$
\begin{gathered}
X=R / d \quad \text { et } X_{0}=\rho_{0} / d . \\
F_{12}=\frac{1}{2}\left(1+\frac{X^{2}-X_{0}^{2}-1}{\sqrt{\left(X^{2}+X_{0}^{2}+1\right)^{2}-4 X^{2} X_{0}^{2}}}\right) .
\end{gathered}
$$

Formule qui redonne bien (21) si $X_{0}=0$.

Nous donnons figure 5 , les courbes représentatives de cette formule, pour quelques valeurs de $X_{0}$, en fonction de $X=R / d$.

Remarque : On a noté que le problème des facteurs de forme pour des surfaces planes et parallèles et pour des dièdres droits sont traités avec des systèmes d'axes différents. Pour des raisons de commodités de calculs, nous avons pris le système de référence au centre des surfaces planes et parallèles et pour les mêmes raisons, il est en limite des surfaces pour les dièdres droits.

Il arrive dans certains cas (surfaces composites), que l'on ait à prendre un même système de référence pour ces deux types de problème. Nous donnons ci-dessous les formules à employer dans le cas le plus courant de la boîte parallélipipèdique.

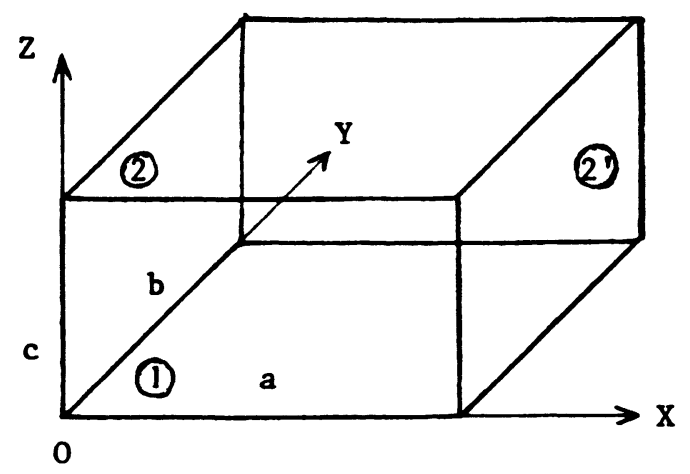




\section{Coordonnées réduites}

$$
X=\frac{a}{c} \quad Y=\frac{b}{c}
$$

On a donc à faire un changement de variables sur la formule (15) et un changement d'axes sur la formule (11) où par ailleurs, nous posons $a=2 a$ et $b=2 b$ afin de simplifier l'écriture.

a) Dièdre droit : $F_{12}$ de 1 vers $2^{\prime}$

$$
\begin{aligned}
F_{12} & =\frac{1}{4 \pi X Y}(F(X, Y)-F(X, 0)-F(0, Y)) \\
F(u, v) & =-4 v \sqrt{1+u^{2}} \operatorname{Arctg} \frac{v}{\sqrt{1+u^{2}}}+4 u v \operatorname{Arctg} \frac{v}{u}+\left(u^{2}-v^{2}+1\right) \operatorname{Ln}\left(1+u^{2}+v^{2}\right)-\left(u^{2}-v^{2}\right) \operatorname{Ln}\left(u^{2}+v^{2}\right)
\end{aligned}
$$

b) Plans rectangulaires parallèles : $F_{12}$ de 1 vers 2

$$
\begin{aligned}
F_{12} & =\frac{1}{\pi X Y}(F(X, Y)-F(X, 0)-F(0, Y)) \\
F(u, v) & =2 u \sqrt{1+v^{2}} \operatorname{Arctg} \frac{u}{\sqrt{1+v^{2}}}+2 v \sqrt{1+u^{2}} \operatorname{Arctg} \frac{v}{\sqrt{1+u^{2}}}-\operatorname{Ln}\left(1+u^{2}+v^{2}\right) .
\end{aligned}
$$

4. Conclusion. - Ainsi que nous l'indiquions au début, nous pensons que le calcul numérique des facteurs de forme, peut se faire de façon simple et précise sur les petites calculatrices programmables modernes, dès lors que les solutions analytiques des cas pratiques les plus courants sont connues. Parmi celles-ci, trois expressions sont plus particulièrement importantes : celles données par les formules (1), (15) et (19). Nous y ajoutons la formule (11), sous la forme (11'), qui correspond à un cas pratique important. Enfin, nous pensons que les formules (9) (18) et (22), donnant les facteurs de forme d'une petite surface émettrice en position quelconque par rapport à la surface réceptive présente un grand intérêt, quoique ces systèmes ne soient pas traités dans les textes spécialisés.

D'autres systèmes à géométrie plus complexe peuvent être envisagés. Malheureusement, on se heurte très vite à l'impossibilité d'obtenir des solutions analytiques. Toutefois, nous pensons que les solutions proposées ici permettent de résoudre sans approximation les cas pratiques les plus courants.

\section{Bibliographie}

[1] Hottel, H. C. et SARofim, A. F., Radiation transfer (Mc Graw-Hill) 1967.

[2] Sacadura, J. F., Rev. Int. Hautes Temp. 8 (1971) 101110.
[3] KREITH, F., Transmission de la chaleur Thermodynamique (Masson) 1967.

[4] SACADURA, J. F., Initiation aux transferts thermiques (Technique et Documentation) 1980. 